PROCEEDINGS OF THE AMERICAN MATHEMATICAL SOCIETY

Volume 125, Number 4, April 1997, Pages 1161-1165

S 0002-9939(97)03717-9

\title{
LAMPERTI-TYPE OPERATORS ON A WEIGHTED SPACE OF CONTINUOUS FUNCTIONS
}

\author{
R. K. SINGH AND BHOPINDER SINGH
}

(Communicated by Palle E. T. Jorgensen)

\begin{abstract}
For a locally convex Hausdorff topological vector space $E$ and for a system $V$ of weights vanishing at infinity on a locally compact Hausdorff space $X$, let $C V_{0}(X, E)$ be the weighted space of $E$-valued continuous functions on $X$ with the locally convex topology derived from the seminorms which are weighted analogues of the supremum norm. A characterization of the orthogonality preserving (Lamperti-type) operators on $C V_{0}(X, E)$ is presented in this paper.
\end{abstract}

\section{INTRODUCTION}

The operators which preserve orthogonality and are order bounded are called Lamperti operators, whereas those preserving only orthogonality are called disjointness preserving operators. We prefer to call the orthogonality preserving operators Lamperti-type operators.

Arendt in Example 2.2 of his paper [3] proves that every Lamperti operator between Banach lattices of complex-valued continuous functions on compact Hausdorff spaces is a weighted composition operator. At the same time, a much more general result characterizing Lamperti-type operators between arbitrary Banach lattices has been proved by Abramovich in [1]. In Theorem 1 of [5], Jamison and Rajagopalan by using the Arendt's result have characterized Lamperti-type operators on the Banach space $C(X, E)$ of continuous functions from a compact Hausdorff space $X$ into a Banach space $E$. A result similar in spirit to that of [5] with $E$ as a locally convex space is proved by the authors in [8]. Recently, Chan [4] has extended the result of [5] to the setting of function modules. More information on the topic can be found in the monograph [2] of Abramovich, Arenson and Kitover.

For a Nachbin system $V$ of weights (non-negative upper semi-continuous functions) on a locally compact Hausdorff space $X$ and for a Hausdorff locally convex topological vector space $E$, let $C V_{0}(X, E)$ denote the weighted space of all continuous functions $f: X \rightarrow E$ such that $v f$ vanishes at infinity on $X$ for every $v \in V$, which is equipped with a Hausdorff locally convex topology derived from the seminorms that are weighted analogues of supremum norm. When $E=\mathbb{C}$, we write

Received by the editors April 14, 1995 and, in revised form, October 23, 1995.

1991 Mathematics Subject Classification. Primary 47B38, 46E40, 47B60.

Key words and phrases. System of weights, weighted space of vector-valued continuous functions, Lamperti operator, strong operator topology, support of a measure.

The second author was supported by NBHM(DAE) Grant No. 40/16/93-G. 
$C V_{0}(X, \mathbb{C})$ as $C V_{0}(X)$. For more details and references on the weighted spaces, we refer to Summers [9], and Singh and Manhas [7].

Let $T$ be a selfmap on $X$ and let $\theta$ be a function on $X$ which takes values either in $\mathbb{C}$ or in $B(E)$, the space of all continuous linear operators on $E$. Then the weighted composition operator on $C V_{0}(X, E)$ induced by the pair $(\theta, T)$ is the continuous linear operator on $C V_{0}(X, E)$, which is denoted by $W_{\theta, T}$ and has the following form:

$$
W_{\theta, T} f(x)=\theta(x)(f(T(x))) \quad \text { for all } f \in C V_{0}(X, E) \text { and } x \in X .
$$

See [7] for details of such operators on the weighted spaces.

In this paper, by using a technique different from [3], [4], we prove (in Theorem 3 below) that every Lamperti-type operator on $C V_{0}(X)$ is a weighted composition operator, for the case when $V$ is $C_{0}^{+}(X)$, the system of all continuous weights vanishing at infinity on $X$. We, then, apply this theorem to obtain a similar result on $C V_{0}(X, E)$.

\section{LAMPERTI-TYPE OPERATORS}

Let us start with the following definition:

Definition 1. Let $f, g \in C V_{0}(X, E)$. Then $f$ and $g$ are called orthogonal (in symbols: $f \perp g$ ) if for each $x \in X$ at least one of the values $f(x)$ or $g(x)$ is zero. A linear operator $A: C V_{0}(X, E) \rightarrow C V_{0}(X, E)$ is Lamperti-type if $A f \perp A g$ whenever $f \perp g$.

In order to present the main result of this paper, we need the following notation and a result from Summers [9]:

Let $E^{*}$ denote the continuous dual of $E, M_{b}(X)$ be the set of all bounded regular Borel measures on $X$, and $K^{+}(X)$ be the system of all constant weights on $X$. For any $f \in C V_{0}(X)$ and any $t \in E$, the function $f_{t}$ defined by setting $f_{t}(x)=f(x) t$ for all $x \in X$ clearly belongs to $C V_{0}(X, E)$. In particular, the constant function $1 \in C V_{0}(X)$ and the constant $t$-function $1_{t} \in C V_{0}(X, E)$ whenever $V$ consists of the weights vanishing at infinity on $X$.

Theorem 2 ([9, Theorem 3.16]). Let $V$ be a Nachbin family on a locally compact Hausdorff space $X$ with $C_{0}^{+}(X) \leq V \leq K^{+}(X)$. Then the map $\Phi: M_{b}(X) \rightarrow$ $C V_{0}(X)^{*}$ defined as $\Phi(\mu) f=\int f d \mu$ for all $f$ in $C V_{0}(X)^{*}$ is a (linear) surjective isomorphism, and in this case we write $C V_{0}(X)^{*}=M_{b}(X)$.

From now on, we work under the assumption that $X$ is a locally compact Hausdorff space, $V=C_{0}^{+}(X)$, and $E$ is a Hausdorff locally convex topological vector space.

Theorem 3. Let $A$ be a continuous linear operator on $C V_{0}(X)$. Then $A$ is Lamperti-type if and only if there exists a selfmap $T$ on $X$ and a function $h$ in $C V_{0}(X)$ such that $A=W_{h, T}$. Moreover, $T$ is uniquely determined and continuous on the set $N(h)=\{x \in X: h(x) \neq 0\}$.

Proof. It is clear that if $A=W_{h, T}$ for some $h$ and $T$, then $A$ is a Lamperti-type operator on $C V_{0}(X)$.

To prove otherwise, suppose that $A$ is a Lamperti-type operator on $C V_{0}(X)$. Let $x \in X$ such that $\mu_{x}=e_{x} \circ A \neq 0$, where $e_{x} \in C V_{0}(X)^{*}$ is the point evaluation at $x\left(e_{x}(f)=f(x)\right.$ for all $f$ in $\left.C V_{0}(X)\right)$. We claim that $\operatorname{spt}\left(\mu_{x}\right)$, the support of $\left|\mu_{x}\right|$, is a singleton set. If this is not true, then let us take distinct points $x_{1}$ and $x_{2}$ 
in $\operatorname{spt}\left(\mu_{x}\right)$, and consider two open sets $G_{1}$ and $G_{2}$ in $X$ with disjoint closures and which also contain $x_{1}$ and $x_{2}$ respectively. Let $f_{1}$ and $f_{2}$ be continuous functions on $X$ such that $-1 \leq f_{i} \leq 0, f_{i}\left(x_{i}\right)=-1$, and $f_{i}\left(X \backslash G_{i}\right)=\{0\}$ for $i=1,2$. Then it is clear that $f_{1}, f_{2} \in C V_{0}(X)$ are orthogonal. Since $\mu_{x} \in C V_{0}(X)^{*}$, there exists a $\mu \in M_{b}(X)$ such that $\Phi(\mu)=\mu_{x}$ (cf. Theorem 2). This implies that

$$
A f(x)=\mu_{x}(f)=\Phi(\mu) f=\int f d \mu
$$

for all $f$ in $C V_{0}(X)$. But, for $f=f_{1}$ and $f=f_{2}$, we have

$$
A f_{1}(x)=\int f_{1} d \mu \geq \int-1 d \mu=(-1) \cdot \mu\left(G_{1}\right) \neq 0
$$

and

$$
A f_{2}(x) \geq(-1) \cdot \mu\left(G_{2}\right) \neq 0
$$

which yields that

$$
\left|A f_{1}(x)\right| \cdot\left|A f_{2}(x)\right| \neq 0 .
$$

This contradiction shows that $\operatorname{spt}\left(\mu_{x}\right)$ is a singleton set.

Let $N=\left\{x \in X: e_{x} \circ A \neq 0\right\}$. Then for each $x$ in $N$, there exists exactly one $T(x)$ in $X$ such that $\operatorname{spt}\left(e_{x} \circ A\right)=\{T(x)\}$. Thus there exists $h(x) \in \mathbb{C} \backslash\{0\}$ such that

$$
e_{x} \circ A=h(x) e_{T(x)} \text { for all } x \text { in } N .
$$

For $x$ outside $N$, let $h(x)=0$ and $T(x)$ be arbitrary. Then we see that

$$
A f(x)=e_{x} \circ A(f)=h(x) e_{T(x)}(f)=h(x) f(T(x))
$$

for all $f$ in $C V_{0}(X)$ and $x$ in $X$. Thus $A=W_{h, T}$. Since $1 \in C V_{0}(X)$, we have $h=A 1 \in C V_{0}(X)$. The fact that

$$
f(T(x))=\frac{A f(x)}{h(x)}
$$

holds for all $f$ in $C V_{0}(X)$ and $x$ in $N(h)$ implies that $T$ must be continuous on the set $N=N(h)$.

Next, applying the technique used in [8], we proceed to obtain a similar version on the vector-value space $C V_{0}(X, E)$.

Suppose $A$ is an operator on $C V_{0}(X, E)$. For each $t$ in $E$ and $t^{*}$ in $E^{*}$, we define the operator $S_{t, t^{*}}$ on $C V_{0}(X)$ as

$$
S_{t, t^{*}} f(x)=t^{*}\left(A f_{t}(x)\right)
$$

for all $f$ in $C V_{0}(X)$ and $x$ in $X$. Then it is clear that $S_{t, t^{*}}$ is Lamperti-type if $A$ is.

Also, for each $x$ in $X$, if we define $\pi(x): E \rightarrow E$ as

$$
\pi(x) t=A 1_{t}(x) \text { for all } t \text { in } E,
$$

then it is easy to see that each $\pi(x)$ is an operator on $E$, and $\pi \in C V_{0}\left(X, B_{s}(E)\right)$, where $B_{s}(E)$ is the space $B(E)$ equipped with the strong operator topology.

Proposition 4. Suppose the operator $S_{t, t^{*}}$ as defined in (1) is Lamperti-type on $C V_{0}(X)$. Then there exist a selfmap $T_{t, t^{*}}$ on $X$ and a function $h_{t, t^{*}}$ in $C V_{0}(X)$ such that

$$
S_{t, t^{*}}=W_{h_{t, t^{*}}, T_{t, t^{*}}}
$$


Moreover, $T_{t, t^{*}}$ is continuous in a neighbourhood of each $x_{0}$ for which $\pi\left(x_{0}\right) t \neq 0$ and, further, $T_{t, t^{*}}$ is independent of $t$ and $t^{*}$.

Proof. It follows from Theorem 3 that $S_{t, t^{*}}$ is a weighted composition operator on $C V_{0}(X)$, and the fact that $T_{t, t^{*}}$ is independent of $t$ and $t^{*}$ can be proved in a similar way as shown in $[5$, p. 310].

Theorem 5. Let $A$ be an operator on $C V_{0}(X, E)$. Then $A$ is a Lamperti-type operator if and only if there exist a selfmap $T$ on $X$ and a function $\pi$ in $C V_{0}\left(X, B_{s}(E)\right)$ such that $T$ is continuous on the set $N(\pi)=\{x \in X: \pi(x) \neq 0\}$ and $A=W_{\pi, T}$.

Proof. Suppose $A=W_{\pi, T}$ for some $\pi$ and $T$. Then it is clear that $A$ is a Lampertitype operator on $C V_{0}(X, E)$.

For the converse part, suppose $A$ is a Lamperti-type operator on $C V_{0}(X, E)$. Then, for each $t$ in $E$ and $t^{*}$ in $E^{*}$, the operator $S_{t, t^{*}}$ as defined in (1) is a Lampertitype operator on $C V_{0}(X)$ and (2) implies the existence of $\pi$ in $C V_{0}\left(X, B_{s}(E)\right)$. According to Proposition 4 there exists a selfmap $T_{t, t^{*}}$ on $X$ and a function $h_{t, t^{*}}$ in $C V_{0}(X)$ such that $T_{t, t^{*}}$ is continuous on $N(\pi)$ and

$$
S_{t, t^{*}} f(x)=h_{t, t^{*}}(x) f(T(x))
$$

for all $f$ in $C V_{0}(X)$, where $T(x)=T_{t, t^{*}}(x)$ for all $x$ in $X$ since $T_{t, t^{*}}$ is independent of $t$ and $t^{*}$, and

$$
h_{t, t^{*}}(x)=S_{t, t^{*}} 1(x)=t^{*}\left(A 1_{t}(x)\right)=t^{*}(\pi(x) t),
$$

which is obtained by using (1) and (2) in (3). Thus we see that

$$
t^{*}\left(A f_{t}(x)\right)=S_{t, t^{*}} f(x)=t^{*}(\pi(x) t) \cdot f(T(x))=t^{*}\left(\pi(x) f_{t}(T(x))\right)
$$

for all $f$ in $C V_{0}(X), t$ in $E, t^{*}$ in $E^{*}$, and $x$ in $N(\pi)$. This fact implies that the following formula is valid:

$$
A f_{t}(x)= \begin{cases}\pi(x)\left(f_{t}(T(x))\right) & \text { for } x \text { in } N(\pi), \\ 0 & \text { otherwise. }\end{cases}
$$

But the closed linear span of the set $\left\{f_{t}: f \in C V_{0}(X), t \in E\right\}$ is dense in $C V_{0}(X, E)$ [6, Theorem 4], so we conclude that (5) is valid for all $f$ in $C V_{0}(X, E)$. This shows that $A=W_{\pi, T}$.

\section{ACKNOWLEDGEMENT}

The authors are thankful to the referee for drawing their attention to the paper [1] of Abramovich and for his valuable suggestions improving the quality of the paper.

\section{REFERENCES}

1. Y. A. Abramovich, Multiplicative representation of disjointness preserving operators, Netherl. Acad. Wetensch. Proc. Ser. A 45 (1983), 265-279. MR 85f: 47040

2. Y. A. Abramovich, E. L. Arenson and A. K. Kitover, Banach $C(K)$-modules and operators preserving disjointness, Pitman Math. Series 277, New York, 1992. MR 94d:47027

3. W. Arendt, Spectral properties of Lamperti operators, Indiana Univ. Math. J. 32 (1983), 199215. MR 85d:47040

4. J. T. Chan, Operators with the disjoint support property, J. Operator Theory 24 (1990), 383391. MR 93c: 47035

5. J. E. Jamison and M. Rajagopalan, Weighted composition operator on $C(X, E)$, J. Operator Theory 19 (1988), 307-317. MR 90b:47052 
6. J. B. Prolla, Weighted approximation of continuous functions, Bull. Amer. Math. Soc. $\mathbf{7 7}$ (1971), 1021-1024. MR 44:7199

7. R. K. Singh and J. S. Manhas, Composition operators on function spaces, North-Holland Math. Studies 179, Amsterdam, 1993. MR 95d:47036

8. R. K. Singh and Bhopinder Singh, Weighted composition operators on a space of vector-valued functions, Far East J. Math. Sci. 2 (1994), 95-100. CMP 95:04

9. W. H. Summers, Weighted locally convex spaces of continuous functions, $\mathrm{Ph}$. D. Thesis, Louisiana State University, 1968.

Department of Mathematics, University of Jammu, Jammu 180 004, India

Current address, Bhopinder Singh: Department of Mathematics, Government College of Engineering and Technology, Jammu 180 001, India 